\title{
Giant Molecular Associations in M51
}

\author{
M. Hitschfeld ${ }^{1}$, C. Kramer ${ }^{1}$, K. Schuster ${ }^{2}$, S. Garcia-Burillo ${ }^{3}$ \\ and J. Stutzki ${ }^{1}$ \\ ${ }^{1}$ KOSMA, Universität zu Köln, Germany; ${ }^{2}$ IRAM, Grenoble, France; ${ }^{3}$ Centro Astronomico \\ de Yebes, Guadalajara, Spain;
}

\begin{abstract}
We present a ${ }^{12}$ CO 2-1 map of M51 (Schuster et al. 2006) at 11" resolution observed with HERA at the IRAM-30m telescope. The map covers the companion galaxy NGC5195 as well as the south-western arm out to $12 \mathrm{kpc}$. Using the IRAM-30m data and the clump finding procedure GAUSSCLUMPS (Stutzki et al. 1990), we obtain the masses, positions, peak temperatures and more intrinsic properties as i.e. deconvolved sizes of Giant Molecular Associations (GMAs) in M51 (Hitschfeld et al. 2007, in prep.).
\end{abstract}

\section{Results}

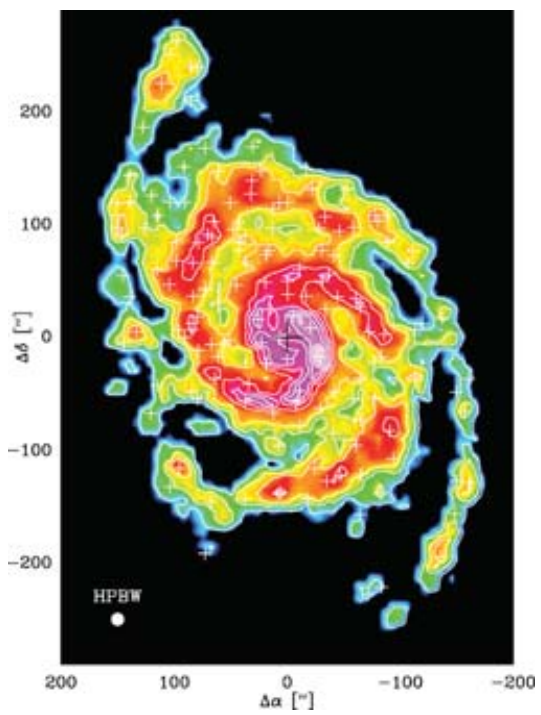

Fig. 1 Map of ${ }^{12} \mathrm{CO} 2-1$ integrated intensities $\left[\mathrm{Kkms}^{-1}\right]$ showing M51 and its companion galaxy NGC 5195 in the northeast. The image has a resolution of $11^{\prime \prime}$ and is constructed from a masked moment calculation. The center positions of the 155 clumps produced by GAUSSCLUMPS are indicated by white crosses. The mass range for the fitted clumps is $4.910^{5} \mathrm{M}_{\odot}$ to $1.210^{8} \mathrm{M}_{\odot}$.

The HERA map of ${ }^{12} \mathrm{CO} 2-1$ (Fig. 1) is the first CO map of M51 encompassing the companion galaxy as well as the south-western arm out to radii of $\sim 12 \mathrm{kpc}$ in a homogeneously sampled data set at linear scales of down to $450 \mathrm{pc}$. We presented a detailed study of the distribution of molecular gas, radial averages of molecular and atomic gas densities, local Schmidt law and gravitational stability in M51 in Schuster et al.(2006). We decompose the ${ }^{12} \mathrm{CO} 2-1$ emission into three-dimensional Gaussian-shaped clumps using GAUSSCLUMPS and obtain i.e. positions, velocities and deconvolved sizes of the clumps.

\section{References}

Schuster, K.-F., Kramer, C., Hitschfeld, M., Garcia-Burillo, S., \& Mookerjea, B. 2006, A\&A submitted

Stutzki, J. \& Güsten, R. 1990, ApJ 356, 513 